\title{
The expression and clinical value of ubiquitin carboxyl-terminal hydrolase L1 in the blood of neonates with hypoxic ischemic encephalopathy
}

\author{
Shuying Zeng, Yubo Huang, Tao Zhong, Tao Huang, Xianyan Dong, Huadong Zhu, Fulian Ouyang \\ Department of Pediatrics, Ganzhou People's Hospital, Ganzhou, China \\ Contributions: (I) Conception and design: S Zeng; (II) Administrative support: S Zeng, F Ouyang; (III) Provision of study materials or patients: F \\ Ouyang; (IV) Collection and assembly of data: All authors; (V) Data analysis and interpretation: All authors; (VI) Manuscript writing: All authors; \\ (VII) Final approval of manuscript: All authors. \\ Correspondence to: Fulian Ouyang. Department of Pediatrics, Ganzhou People’s Hospital, Ganzhou 341000, China. Email: ouyangfulian0705@sina.com.
}

\begin{abstract}
Background: Neonatal hypoxic ischemic encephalopathy (HIE) can result in mental retardation due to the associated brain damage. Early identification of brain injury is vital for the prevention and treatment of brain damage in neonates. This study investigated the expression levels of serum ubiquitin carboxy-terminal hydrolase L1 (UCH-L1) in neonates with HIE and its correlation with brain damage.
\end{abstract}

Methods: From January 2019 to December 2020, 56 cases of neonatal patients with HIE were selected as the observation group, and 60 cases of healthy newborns delivered in our hospital during the same period were selected as the control group. Blood samples were obtained from neonates and the serum expression of UCH-L1 was detected by enzyme-linked immunosorbent assays (ELISAs). The relationship between UCH-L1 and neonatal prognosis and clinical features was analyzed.

Results: Compared with the healthy control group, the serum levels of UCH-L1 in the observation group was significantly higher $(2.28 \pm 1.21$ vs. $0.81 \pm 0.39 \mathrm{ng} / \mathrm{mL}, \mathrm{P}=0.000)$. Furthermore, at 6 hours after birth, the serum levels of UCH-L1 were significantly higher in neonates with moderate to severe HIE compared to patients with mild HIE $(2.92 \pm 0.80$ and $1.76 \pm 0.72 \mathrm{ng} / \mathrm{mL}$, respectively, $\mathrm{P}=0.000)$. Pearson correlation analysis showed that the expression levels of UCH-L1 were negatively correlated with the development quotient (DQ), intelligence index (MI), and the Neonatal Behavioral Neurological Assessment (NBNA) score of HIE newborns $(\mathrm{P}<0.05)$.

Conclusions: The level of UCH-L1 protein expression is elevated in the serum of newborns with HIE, and this may have a certain clinical value in predicting the intelligence of children.

Keywords: Ubiquitin carboxy-terminal hydrolase L1 (UCH-L1); hypoxic ischemic encephalopathy (HIE); newborn; clinical value

Submitted Jun 16, 2021. Accepted for publication Aug 01, 2021.

doi: $10.21037 / \mathrm{tp}-21-327$

View this article at: https://dx.doi.org/10.21037/tp-21-327

\section{Introduction}

Neonatal hypoxic ischemic encephalopathy (HIE) is one of the most common diseases in pediatrics. HIE has a complicated pathogenesis and is usually triggered by intrauterine distress or neonatal asphyxia during the perinatal period. Clinical signs of HIE includes drowsiness, irritability, and coma. Surviving neonates with HIE are often characterized by delayed development, cerebral palsy, and even death (1-3). Therefore, biomarkers that can detect early intracranial injury is crucial. In the last decade, research has shown that acute brain injury is characterized by the presence of ubiquitin carboxy-terminal hydrolase L1 (UCH-L1) (4,5). In neonates with HIE, early stage ischemia and hypoxia result in different degrees of acute 
Table 1 A comparison of the general patient characteristics

\begin{tabular}{lcccc}
\hline Variable & Observation group $(\mathrm{n}=56)$ & Control group $(\mathrm{n}=60)$ & $\mathrm{t} / \chi^{2}$ value & $\mathrm{P}$ value \\
\hline Gender (male/female) & $26 / 30$ & $32 / 28$ & 0.552 & 0.457 \\
Gestational age (weeks) & $38.51 \pm 1.45$ & $38.47 \pm 1.13$ & 0.166 & 0.868 \\
Head circumference $(\mathrm{cm})$ & $33.65 \pm 3.46$ & $34.53 \pm 3.74$ & 1.313 & 0.192 \\
Weight $(\mathrm{kg})$ & $3.25 \pm 0.43$ & $3.32 \pm 0.45$ & 0.855 & 0.394 \\
\hline
\end{tabular}

brain injury. Thus, UCH-L1 may be a promising biomarker of brain damage in neonates with HIE and may be a useful predictor of later neurodevelopmental outcomes. This report investigated the significance of UCH-L1 in the early diagnosis of HIE. We present the following article in accordance with the MDAR reporting checklist (available at https://dx.doi.org/10.21037/tp-21-327).

\section{Methods}

\section{Patients}

A total of 56 neonates with HIE treated in our hospital from January 2019 to December 2020 were enrolled in this study as the observation group. Neonates were included in this study if they were delivered in our hospital and met the diagnostic criteria for neonatal HIE. Patients with infectious diseases of the nervous system, congenital metabolic diseases, or cerebral infarction were excluded. A total of 60 healthy newborns delivered in our hospital during the same period were randomly selected for the control group. All procedures performed in this study involving human participants were in accordance with the Declaration of Helsinki (as revised in 2013). The study was approved by institutional ethics board of the Ganzhou People's Hospital (No.: 2019-142) and informed consent was taken from all the patients.

Out of the 56 neonates in the observation group, there were 26 males and 30 females. The average gestational age was $38.51 \pm 1.45$ weeks, and the average age was $3.25 \pm 2.73$ days. The average head circumference was $33.65 \pm 3.46 \mathrm{~cm}$, and the average weight was $3.25 \pm 0.43 \mathrm{~kg}$. There were 31 neonates with mild HIE and 25 patients with moderate or severe HIE. The control group consisted of 32 males and 28 females. The average gestational age was $38.47 \pm 1.13$ weeks. The average head circumference was $34.53 \pm 3.74 \mathrm{~cm}$, and the average weight was $3.32 \pm 0.45 \mathrm{~kg}$. There were no statistical significances in gender, gestational age, head circumference, weight, age, or any other general data between the two groups
$(\mathrm{P}>0.05 ;$ Table 1).

\section{Observation indicators and detection methods}

To detect UCH-L1 expression, peripheral venous blood samples $(2 \mathrm{~mL})$ were obtained from all subjects at 6 hours after birth. After centrifugation at 3,000 rpm for 15 minutes, the supernatant was retained and the level of UCH-L1 expression in the serum was detected by enzyme-linked immunosorbent assays (ELISAs). The UCH-L1 ELISA kit was purchased from Ray Biotech Inc. (Ray Biotech, Atlanta Science Park, USA) and performed strictly according to the manufacturer's instructions.

To examine the clinical prognosis of the patients, the neuropsychological development of neonates in the observation group was evaluated by the development quotient (DQ) and the intelligence index (MI) according to the Developmental Screening Test. In addition, the Neonatal Behavioral Neurological Assessment (NBNA) score was used to evaluate the neurobehavioral development of newborns. The time points for assessment were 3, 14, and 28 days after birth.

\section{Statistical analysis}

All statistical analyses were performed using SPSS 26.0 software (IBM, Chicago, USA). A two-tailed P value $<0.05$ was considered statistically significant. Enumeration data were analyzed by the $\chi^{2}$ test. Measurement data are presented as mean \pm standard deviation (xxa). Comparison between two groups was performed using the $t$-test, while comparison of multiple groups was conducted using the $\mathrm{F}$ test. The correlation between two measurement data was analyzed using the Pearson correlation analysis.

\section{Results}

\section{A comparison of serum UCH-L1 levels in the two groups}

At 6 hours after birth, the serum levels of UCH-L1 in 
Table 2 Serum levels of ubiquitin carboxy-terminal hydrolase L1 (UCH-L1)

\begin{tabular}{lcccc}
\hline & Observation group $(\mathrm{n}=56)$ & Control group $(\mathrm{n}=60)$ & $\mathrm{t}$ value & $\mathrm{P}$ value \\
\hline $\mathrm{UCH}-\mathrm{L} 1(\mathrm{ng} / \mathrm{mL})$ & $2.28 \pm 1.21$ & $0.81 \pm 0.39$ & 8.929 & 0 \\
\hline
\end{tabular}

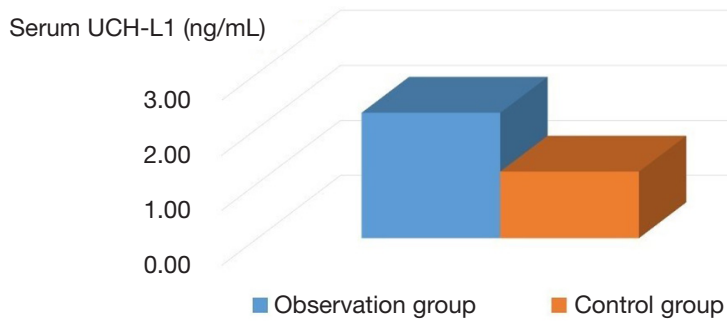

Figure 1 Serum levels of ubiquitin carboxy-terminal hydrolase L1 (UCH-L1) in neonates.

Table 3 Serum levels of ubiquitin carboxy-terminal hydrolase L1 (UCH-L1) in children with different degrees of hypoxic ischemic encephalopathy (HIE)

\begin{tabular}{lcccc}
\hline & Mild HIE $(\mathrm{n}=31)$ & Moderate to severe HIE $(\mathrm{n}=25)$ & $\mathrm{t}$ value & $\mathrm{P}$ value \\
\hline $\mathrm{UCH}-\mathrm{L} 1(\mathrm{ng} / \mathrm{mL})$ & $1.76 \pm 0.72$ & $2.92 \pm 0.80$ & 5.704 & 0 \\
\hline
\end{tabular}

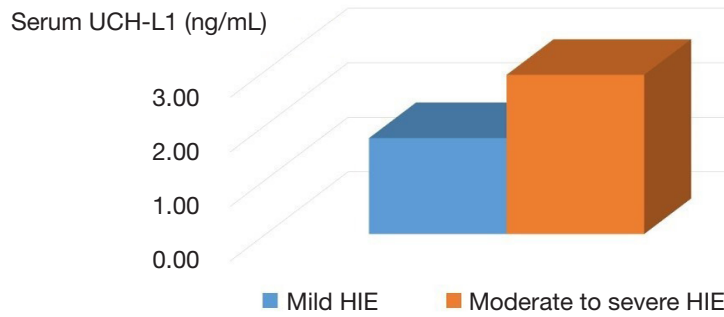

Figure 2 Serum levels of ubiquitin carboxy-terminal hydrolase L1 (UCH-L1) in neonates with different degrees of hypoxic ischemic encephalopathy (HIE).

neonates with HIE was significantly higher than the levels observed in healthy neonates $(2.28 \pm 1.21$ and $0.81 \pm 0.39 \mathrm{ng} / \mathrm{mL}$, respectively; $\mathrm{P}=0.000$; Table 2; Figure 1).

\section{A comparison of serum levels of UCH-L1 in children with different degrees of HIE}

At 6 hours after birth, the levels of serum UCH-L1 in children with moderate to severe HIE was significantly higher than the levels observed in neonates with mild HIE $(2.92 \pm 0.80$ and $1.76 \pm 0.72 \mathrm{ng} / \mathrm{mL}$, respectively, $\mathrm{P}=0.000$;
Table 3; Figure 2).

\section{Clinical prognosis of neonates at follow-up}

At the 6 months follow-up, the Developmental Screening Tests were performed on the neonates. The DQ, MI, and NBNA scores at different time points in the moderate to severe HIE group were significantly lower than those in the mild HIE group and the control group $(\mathrm{P}<0.05)$. The DQ, MI, and NBNA scores in the mild HIE group were also significantly lower compared to the control group 
Table 4 Clinical prognosis of neonates at the 6 months follow-up

\begin{tabular}{lcccc}
\hline Variable & Control group $(\mathrm{n}=60)$ & Mild HIE $(\mathrm{n}=31)$ & Moderate to severe HIE $(\mathrm{n}=25)$ & $\mathrm{F}$ value \\
\hline DQ & $92.68 \pm 5.62$ & $88.52 \pm 5.53^{\mathrm{a}}$ & $72.33 \pm 5.72^{\mathrm{ab}}$ & 9.765 \\
MI & $93.24 \pm 3.79$ & $88.55 \pm 5.03^{\mathrm{a}}$ & $74.24 \pm 5.33^{\mathrm{ab}}$ & 10.024 \\
NBNA score & & & \\
$3 \mathrm{~d}$ & $37.98 \pm 0.82$ & $37.32 \pm 0.76^{\mathrm{a}}$ & $36.32 \pm 0.80^{\mathrm{ab}}$ & 8.949 \\
$14 \mathrm{~d}$ & $38.34 \pm 0.65$ & $37.91 \pm 0.58^{\mathrm{a}}$ & $37.34 \pm 0.52^{\mathrm{ab}}$ & 0 \\
$28 \mathrm{~d}$ & $38.82 \pm 0.62$ & $38.03 \pm 0.69^{\mathrm{a}}$ & $37.54 \pm 0.59^{\mathrm{ab}}$ & 0 \\
\hline
\end{tabular}

${ }^{a}, \mathrm{P}<0.05$, compared with the control group; ${ }^{b}, \mathrm{P}<0.05$, compared with the mild HIE group. HIE, hypoxic ischemic encephalopathy; DQ, development quotient; MI, intelligence index; NBNA, Neonatal Behavioral Neurological Assessment.

Table 5 The correlation between ubiquitin carboxy-terminal hydrolase L1 expression and development quotient and intelligence index neonates with hypoxic ischemic encephalopathy

\begin{tabular}{lcc}
\hline Variable & r value & P value \\
\hline DQ & -0.452 & 0 \\
MI & -0.446 & 0.001 \\
NBNA score & & \\
3 d & -0.413 & 0.002 \\
$14 d$ & -0.462 & 0 \\
28 d & -0.398 & 0.01 \\
\hline
\end{tabular}

$D Q$, development quotient; MI, intelligence index; NBNA, Neonatal Behavioral Neurological Assessment.

$(\mathrm{P}<0.05 ;$ Table 4).

\section{The correlation between UCH-L1 expression and the development quotient and intelligence index in neonates with HIE}

Pearson correlation analysis showed that UCH-L1 expression was negatively correlated with DQ, MI, and NBNA scores in HIE neonates $(\mathrm{P}<0.05$; Table 5$)$.

\section{Discussion}

Brain injury associated with neonatal HIE can be severe, and its sequelae include mental retardation, developmental retardation, and epilepsy (6). Approximately $0.3 \%$ of newborns with HIE develop cerebral ischemia and hypoxic injury, and about $15 \%$ of children with HIE present with varying degrees of sequelae $(7,8)$. At present, the diagnosis of HIE relies heavily on computed tomography (CT), magnetic resonance imaging (MRI), and other imaging modalities, which are relatively subjective. Therefore, the development of potential serum biomarkers is vital for the diagnosis of HIE. UCH-L1 hydrolyzes the chain between ubiquitin and substrate protein, and then the substrate enters the proteasome and is degraded. $\mathrm{UCH}-\mathrm{L} 1$ is a protein distinctly expressed in brain cells, and its levels are related to the degree of neuronal brain damage and the destruction of the blood-brain barrier, which is indicative of nervous system injury (9-11).

This study demonstrated that serum levels of UCH-L1 were significantly elevated in children with HIE (the observation group) compared with neonates in the healthy control group at 6 hours after birth. Furthermore, the levels of UCH-L1 in neonates with moderate to severe HIE were significantly higher than that in neonates with mild HIE at 6 hours after birth. These results are consistent with earlier studies $(12,13)$. Previous work in rat models have shown that the concentration of UCH-L1 increases in the presence of brain damage (14), and the elevation in UCH-L1 levels reaches a peak at 6 hours after birth in brain-damaged human neonates (15). During HIE, brain injury results in the release of inflammatory factors, excessive production of free radicals, and other related phenomena, which interact with each other, leading to degeneration, necrosis, and apoptosis of brain neurons and the destruction of the blood-brain barrier (16-18). At this time, the brain releases a large amount of UCH-L1 protein which enters the blood through the damaged blood-brain barrier, and can thus be detected in the cerebrospinal fluid or blood during the acute stage of brain injury $(19,20)$.

This study also revealed that the serum levels of UCH-L1 in neonates with moderate to severe HIE was significantly higher than that in neonates with mild HIE 
$(\mathrm{P}=0.000)$ at 6 hours after birth. Pearson correlation analysis showed that the levels of UCH-L1 were significantly negatively correlated with DQ, MI, and NBNA score $(\mathrm{P}<0.05)$. The results indicated that the levels of UCH-L1 increase with the progression of the disease, and the higher its concentration, the poorer the prognosis.

In conclusion, the expression levels of UCH-L1 protein can be used for the diagnosis of acute brain injury caused by HIE, and can reflect the severity of HIE and predict the prognosis of children with HIE. Therefore, serum levels of UCH-L1 may be a potential biomarker in clinical practice. We need further studies to investigate whether inhibition of UCH-L1 expression could attenuate brain injury in neonates with HIE or not.

\section{Limitations}

Limited to ethical requirements, we could not detect the expression of UCH-L1 in the brain.

\section{Acknowledgments}

Funding: None.

\section{Footnote}

Reporting Checklist: The authors have completed the MDAR reporting checklist. Available at https://dx.doi. org/10.21037/tp-21-327

Data Sharing Statement: Available at https://dx.doi. org/10.21037/tp-21-327

Conflicts of Interest: All authors have completed the ICMJE uniform disclosure form (available at https://dx.doi. org/10.21037/tp-21-327). The authors have no conflicts of interest to declare.

Ethical Statement: The authors are accountable for all aspects of the work in ensuring that questions related to the accuracy or integrity of any part of the work are appropriately investigated and resolved. All procedures performed in this study involving human participants were in accordance with the Declaration of Helsinki (as revised in 2013). The study was approved by institutional ethics board of the Ganzhou People's Hospital (No.: 2019-142) and informed consent was taken from all the patients.
Open Access Statement: This is an Open Access article distributed in accordance with the Creative Commons Attribution-NonCommercial-NoDerivs 4.0 International License (CC BY-NC-ND 4.0), which permits the noncommercial replication and distribution of the article with the strict proviso that no changes or edits are made and the original work is properly cited (including links to both the formal publication through the relevant DOI and the license). See: https://creativecommons.org/licenses/by-nc-nd/4.0/.

\section{References}

1. Domínguez-Dieppa F, Cardetti M, Rodríguez S, et al. Hypoxic Ischemic Encephalopathy in Units Reporting to the Ibero-American Society of Neonatology Network: Prevalence and Mortality. MEDICC Rev 2021;23:30-4.

2. Ogawa Y, Tsuji M, Tanaka E, et al. Bone Morphogenetic Protein (BMP)-3b Gene Depletion Causes High Mortality in a Mouse Model of Neonatal Hypoxic-Ischemic Encephalopathy. Front Neurol 2018;9:397.

3. Dukhovny D, Zupancic JAF. Longer and deeper cooling for hypoxic ischemic encephalopathy in neonates does not reduce mortality. Acta Paediatr 2017;106:1200.

4. Mrozek S, Delamarre L, Capilla F, et al. Cerebral Expression of Glial Fibrillary Acidic Protein, Ubiquitin Carboxy-Terminal Hydrolase-L1, and Matrix Metalloproteinase 9 After Traumatic Brain Injury and Secondary Brain Insults in Rats. Biomark Insights 2019;14:1177271919851515.

5. Blyth BJ, Farahvar A, He H, et al. Elevated serum ubiquitin carboxy-terminal hydrolase L1 is associated with abnormal blood-brain barrier function after traumatic brain injury. J Neurotrauma 2011;28:2453-62.

6. Umran RM, Al-Tahir M, Jagdish D, et al. Insulin-Like Growth Factor-1 Levels in Term Newborns with HypoxicIschemic Encephalopathy. Am J Perinatol 2016;33:640-5.

7. Palsdottir K, Dagbjartsson A, Thorkelsson T, et al. Birth asphyxia and hypoxic ischemic encephalopathy, incidence and obstetric risk factors. Laeknabladid 2007;93:595-601.

8. Thorngren-Jerneck K, Ohlsson T, Sandell A, et al. Cerebral glucose metabolism measured by positron emission tomography in term newborn infants with hypoxic ischemic encephalopathy. Pediatr Res 2001;49:495-501.

9. Anderson TN, Hinson HE. Damaged: Elevated GFAP and UCH-L1 as the Black Flag of Brain Injury. Resuscitation 2020;154:110-1.

10. Papa L, Zonfrillo MR, Welch RD, et al. Evaluating glial and neuronal blood biomarkers GFAP and UCH-L1 as 
gradients of brain injury in concussive, subconcussive and non-concussive trauma: a prospective cohort study. BMJ Paediatr Open 2019;3:e000473.

11. Morris MC, Bercz A, Niziolek GM, et al. UCH-L1 is a Poor Serum Biomarker of Murine Traumatic Brain Injury After Polytrauma. J Surg Res 2019;244:63-8.

12. Ramezani F, Bahrami-Amiri A, Babahajian A, et al. Ubiquitin C-Terminal Hydrolase-L1 (UCH-L1) in Prediction of Computed Tomography Findings in Traumatic Brain Injury; a Meta-Analysis. Emerg (Tehran) 2018;6:e62.

13. Shahjouei S, Sadeghi-Naini M, Yang Z, et al. The diagnostic values of UCH-L1 in traumatic brain injury: A meta-analysis. Brain Inj 2018;32:1-17.

14. Huang XJ, Glushakova O, Mondello S, et al. Acute Temporal Profiles of Serum Levels of UCH-L1 and GFAP and Relationships to Neuronal and Astroglial Pathology following Traumatic Brain Injury in Rats. J Neurotrauma 2015;32:1179-89.

15. Mir IN, Steven Brown L, Rosenfeld CR, et al. Placental clearance/synthesis of neurobiomarkers GFAP and $\mathrm{UCH}-\mathrm{L} 1$ in healthy term neonates and those with moderate-severe neonatal encephalopathy. Pediatr Res 2019;86:500-4.

16. Richard M, Lagares A, Bondanese V, et al. Study protocol

Cite this article as: Zeng S, Huang Y, Zhong T, Huang T, Dong X, Zhu H, Ouyang F. The expression and clinical value of ubiquitin carboxyl-terminal hydrolase L1 in the blood of neonates with hypoxic ischemic encephalopathy. Transl Pediatr 2021;10(8):2063-2068. doi: 10.21037/tp-21-327 for investigating the performance of an automated blood test measuring GFAP and $\mathrm{UCH}-\mathrm{L} 1$ in a prospective observational cohort of patients with mild traumatic brain injury: European BRAINI study. BMJ Open 2021;11:e043635.

17. Liu $\mathrm{H}$, Rose $\mathrm{ME}, \mathrm{Ma} \mathrm{X}$, et al. In vivo transduction of neurons with TAT-UCH-L1 protects brain against controlled cortical impact injury. PLoS One 2017;12:e0178049.

18. Zaigham M, Lundberg F, Hayes R, et al. Umbilical cord blood concentrations of ubiquitin carboxyterminal hydrolase L1 (UCH-L1) and glial fibrillary acidic protein (GFAP) in neonates developing hypoxicischemic encephalopathy. J Matern Fetal Neonatal Med 2016;29:1822-8.

19. Rhine T, Babcock L, Zhang N, et al. Are UCH-L1 and GFAP promising biomarkers for children with mild traumatic brain injury? Brain Inj 2016;30:1231-8.

20. Papa L, Brophy GM, Welch RD, et al. Time Course and Diagnostic Accuracy of Glial and Neuronal Blood Biomarkers GFAP and UCH-L1 in a Large Cohort of Trauma Patients With and Without Mild Traumatic Brain Injury. JAMA Neurol 2016;73:551-60.

(English Language Editor: J. Teoh) 